\title{
A Case of Successful Pregnancy Outcome with a Large Ovarian Cyst- A Case Report
} Janna $\mathrm{JR}^{1}$, Khanam $\mathrm{K}^{2 *}$

\begin{abstract}
Ovarian cysts during pregnancy are not uncommon. With increasing use of early antenatal ultrasound, the incidental discovery of adnexal masses during early gestation is a clinically relevant problem that requires careful consideration.

Here, we have presented a case in pregnancy with a large ovarian cyst with a history of unilateral oophorectomy in pre-pregnancy state due to complex ovarian mass.

She is a case of 26 years old primigravid, presented at 8 weeks of gestation with clinical and ultasonographic detection of a large cyst (about $9.2 \mathrm{~cm} \mathrm{x} 6.9 \mathrm{~cm}$ ) in the left ovary with suspicion of dermoid cyst. She had a history of right sided oophorectomy due to complex ovarian cyst at her 18 years of age which was histologically proved to be benign. This patient was treated conservatively with relevant investigation and tumour markers which was found insignificant with an aim to continue pregnancy until at least 14 weeks of gestation and was vigilant enough for early detection of any complication. Operative interference was not done at this stage due to increased chance of early fetal loss $\&$ clinically benign nature of the tumour. The tumour was persisting and gradually increasing in nature and at 11 weeks gestation it was about $11 \mathrm{~cm} \times 10 \mathrm{~cm}$, suggestive of dermoid. Although clinically stable, with a fairly large ovarian cyst which was increasing in size \& with a suspicion of future torsion that will damage the only persisting ovary, this patient underwent laparotomy followed by ovarian cystectomy at 15 weeks of gestation. Close monitoring of the rest of the pregnancy period was done $\&$ it was uneventful except at 32 weeks of gestation she developed PIH which was managed conservatively. She underwent caesarean section at 38 weeks of gestation due to fetal distress.
\end{abstract}

\section{Keywords}

Ovarian cyst, Pregnancy, Caesarean section.

\section{Introduction}

The overall estimated incidence of adnexal masses in pregnancy ranges from $(10 \%$ to $25 \%){ }^{1}$ The incidence of ovarian cyst in pregnancy has increased with the use of dating ultrasound in the first trimester. It enhances anxiety to the pregnant women as it involves increased investigation, admission and antenatal visits. ${ }^{2}$
However, as gestational age advances, the incidence of adnexal masses gradually decreases likely secondary to spontaneous resolution of many of these masses. ${ }^{3}$

The most common ovarian masses in premenopausal women are functional cyst like a follicular or corpus luteum, are due to hormonal influence. On the other hand, dermoid cyst are the most 
the most common nonfunctional ovarian masses in premenopausal women. ${ }^{4,5,6}$ Observational studies evaluating sonographically detectable adnexal pathology during pregnancy estimate an incidence of $1 \%-4 \%$ with the majority of masses resolving spontaneously. ${ }^{7,8}$

Asymptomatic and benign cysts can be managed conservatively while persistent symptomatic ovarian cysts are at increased risk of torsion, rupture and rarely obstructing labour and few cases with a typical feature may represent with malignancy. In these instances, surgical intervention may be done. ${ }^{2}$

The timing of surgical interventions for adnexal pathology during pregnancy also requires careful consideration. There is evidence to support the ideal time for intervention is 14 to 22 weeks of gestational age. ${ }^{3}$ However at any time, if there is evidence of torsion, rupture or malignancy immediate surgery is required. ${ }^{9}$

Here in we present a case of a fairly large ovarian cyst in a pregnant female who had a history of pre-pregnancy oophorectomy for complex ovarian mass and its management a with a successful pregnancy outcome.

\section{Case Report}

A 26-year-old female married since last 4 years, primigravid was booked in our antenatal outpatient department at 8 weeks of gestation with mild lower abdominal discomfort. On examination, her vital signs were stable. Per abdomen revealed no tenderness and guarding but a fairly large mass, central and deviated to left side movable in all direction about $10 \mathrm{~cm} \times 10 \mathrm{~cm}$ was found. Ultrasound showed a single live intrauterine gestation corresponding to 8 weeks of gestation with a large cyst with internal echogenic mass measuring about $9.2 \mathrm{~cm} \times 6.9 \mathrm{~cm}$ sized seen in the left ovary suggestive of dermoid cyst. Her basic laboratory investigations were normal. Tumour marker showed moderately increased CA-125 \& CA-19-9 with other markers such as CEA, B-hCG, CA 15-3 within normal limit. Colour Doppler ultrasonography showed no other intra-abdominal pathology with same sized ovarian mass without any vascular aberration. This patient had a history of right sided oophorectomy for complex ovarian mass at her 18 years of age which proved histologically benign.

Patient was managed inpatient conservatively with supportive treatment with analgesic \& progesterone support with an aim to continue pregnancy. Within few days she was discharged with good condition after exclusion of all other pathology with a weekly follow-up at ante-natal clinic. She was reasonably well until 11 weeks of gestation when she presented with lower abdominal discomfort. Clinically she was stable. Ultrasonography showed a left dermoid about $11 \mathrm{~cm} \times 10 \mathrm{~cm}$ with 11 weeks live single intrauterine pregnancy with no feature of torsion or rupture. Basic investigations were normal. Repeat tumour marker showed normal findings. Our aim was to continue pregnancy until at least 14 weeks of gestation with close monitoring and supportive treatment. She was complaining of on \& off lower abdominal discomfort. Although clinically stable, with a fairly large ovarian cyst which was increasing in size \& with a suspicion of future torsion that will damage the only existing ovary, she underwent laparotomy at 15 
weeks of gestation. Per-operatively there was a left sided ovarian cyst about $12 \mathrm{~cm} \times 10 \mathrm{~cm}$ in size. Left tube was adherent to ovary. Rightsided ovary was absent. There was no ascitic fluid. Left ovarian cystectomy was done with preservation and reconstruction of ovarian tissue as far as possible. The post- operative period was uncomplicated and patient discharged after a week. Histopathology of the cyst was mature cystic teratoma (dermoid cyst). Patient was advised to follow-up regularly in antenatal clinic.

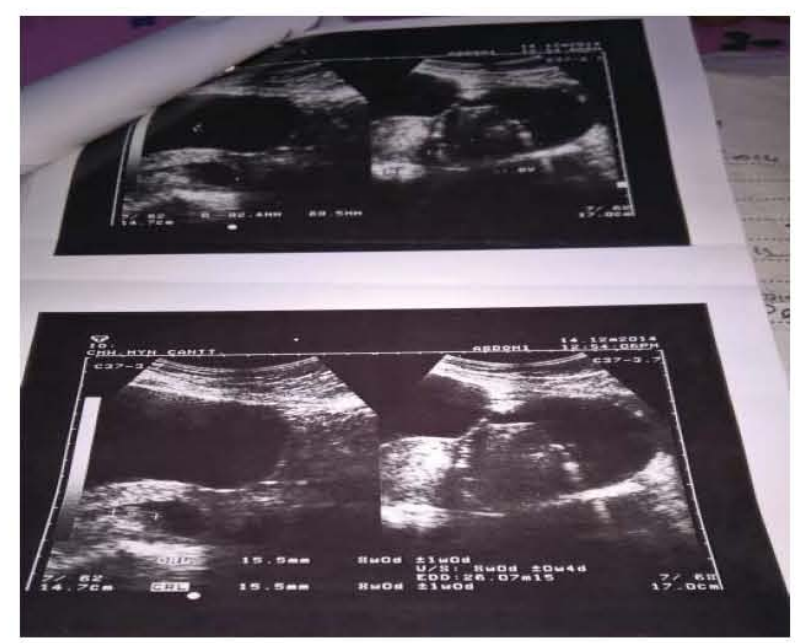

Fig. 1: $9.2 \mathrm{~cm} \times 6.9 \mathrm{~cm}$ ovarian cyst at 8 weeks.

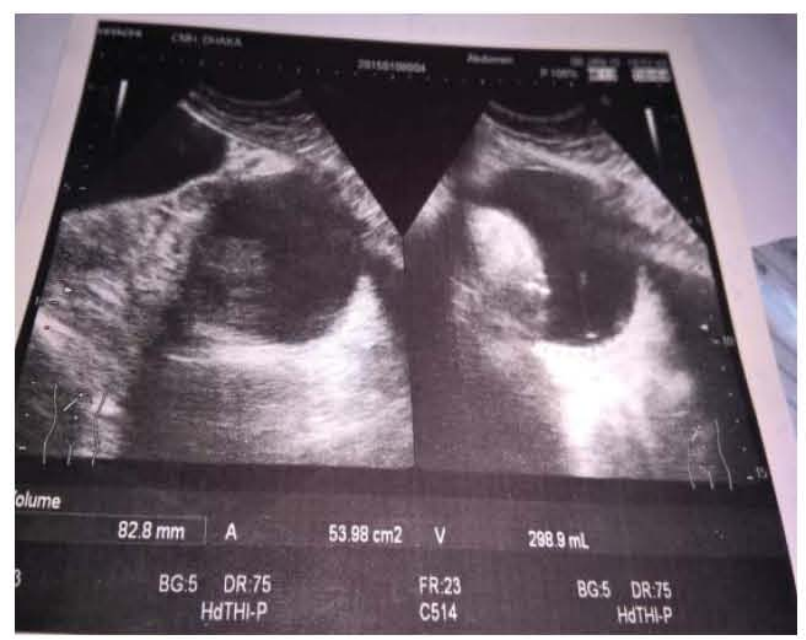

Fig. 2: $11 \mathrm{~cm} \times 10 \mathrm{~cm}$ ovarian cyst at 11 weeks of gestation.
Her subsequent antenatal period was uneventful except she developed $\mathrm{PIH}$ at about 32 weeks of gestation. She was managed conservatively with close monitoring, regular growth scan, related investigation which showed no abnormality. Patient admitted at 37 weeks 5 days of pregnancy with Less foetal movement (LFM) and $\mathrm{PIH}$. She underwent caesarean section at 38 weeks of gestation for foetal distress with PIH. She delivered a healthy male baby of $2.7 \mathrm{~kg}$, two turns of cord were tightly encircled the neck. Per-operatively right ovary was found absent with left ovary small in size but healthy looking. Post-operative period was uneventful and patient discharged with good condition.

\section{Discussion}

The frequency of adnexal mass in pregnancy ranges from ( $2 \%$ to $10 \%) .{ }^{1}$ The most common types are dermoid (25\%), corpus luteal cyst, functional cyst, paraovarian (17\%), serous cystadenoma (14\%), mucinous cystadenoma (11\%), endometrioma ( $8 \%)$, carcinoma $(2.8 \%)$, low malignant potential tumour (3\%) and leiyomyoma (2\%). ${ }^{6}$

Ultrasound serves a dual purpose during pregnancy complicated by adnexal masses, one is to characterize the mass and the second is to provide diagnostic assessment in the acute setting and it is also used as an adjunct to clinical evaluation to rule out ovarian torsion when patients present with abdominal pain and ovarian mass. ${ }^{9,10,11}$

The woman in this case presented with both clinical and ultrasonographically diagnosed case of fairly large ovarian mass with suspicion of dermoid cyst. Ovarian torsion was ruled out both clinically and by ultrasonography. 
The tumour markers like CA-125, B-hCG are insignificant in diagnosis as they are usually raised in pregnancy. However, they can be used for follow up in tumour control. ${ }^{5}$

In this case tumour markers was done at initial presentation and also for follow-up purpose.

Asymptomatic and benign cyst can be managed conservatively while persistent symptomatic ovarian cysts are at increased risk of torsion, rupture. In these cases surgical intervention can be done $\&$ there is evidence to support the ideal time for intervention is 14 to 22 weeks of gestation. ${ }^{2,3}$ In a retrospective review of surgical intervention for adnexal pathology, $12 \%$ of women had preterm delivery, $3.3 \%$ experienced spontaneous abortions and there were 3 perinatal deaths among 60 infants, 2 of which for congenital anomalies. However Whitecar et al found adverse fetal outcomes including preterm delivery and fetal loss were significantly less frequent if surgery occurred prior to 23 weeks gestation. ${ }^{13,14}$

Histologically the majority of adnexal masses in pregnancy are benign. The most common histological diagnoses are dermoid cyst (37$50 \%$ ), cystadenoma (20-24\%), endometrioma (5-11\%), and functional cysts (6-13\%). $5,15,16$

In this present case although the women presented with a fairly large ovarian cyst in early pregnancy, she was managed conservatively until 15 weeks of gestation. With the suspicion of torsion \& gradual increased in size laparotomy followed by ovarian cystectomy was done.

In our case the histopathology of the cyst was mature cystic teratoma (dermoid cyst).
Her remaining antenatal period was uneventful except development of PIH which was managed accordingly. She delivered at term pregnancy at 38 weeks gestation due to foetal distress with good foetal outcome.

\section{Conclusion}

In summary, the incidence of ovarian cyst has increased with increased use of dating ultrasonography. Prior to the use of routine first trimester ultrasound, adnexal mass only become clinically relevant if symptomatic or if they are large enough to palpate on physical examination. Knowing that the majority of ovarian cysts in pregnancy are benign and resolve spontaneously, management is usually conservative with serial ultrasound monitoring \& other laboratory investigation.

So, decision for surgical management should outweigh the risks of adverse perinatal outcome. In these regard it is best to avoid surgery in the first trimester and ideal time for intervention is 14 to 22 weeks of gestation.

With proper planning, management and expertise the pregnant women with ovarian cyst can have a good maternal and fetal outcome.

\section{References}

1. Schwartz N, Timor-Tritsch I. E, and Wang E. Adnexal masses in pregnancy. Clin Obstet Gynecol. 2009; 52(4): 570-85.

2. Wang PH, Chang WH, Cheng MH, Horng HC. Management of adnexal masses during pregnancy. J Obstet Gynaecol Ros. 2009; 35(3): 597-8.

5. Parsons $\mathbf{A ~ K}$. Imaging the human corp us luteum. J Ultrasound Med. 2001; 20(8):811-9.

6. Giuntoli RL 2nd, Vang RS, Bristow RE. Evaluation and management of adnexal masses during pregnancy. Clin Obstet Gynecol. 2006; 49(3): 492-505. 
7. Hoover $\mathrm{K}$, Jenkins TR. Evaluation and management of adnexal mass in pregnancy. Am J Obstet Gynecol, 2011; 205(2):97-102.

8. Nelson MJ, Cavalieri R, Graham D, Sanders RC, et al., Cysts in Pregnancy discovered by sonography. J Clin ultrasound. 1986; 14(7): 509-12.

9. Hill LM, Connors-Beatty DJ, Nowak A, Tush B. The role of ultrasonography in the detection and management of adnexal masses during the second and third trimesters of pregnancy. Am J Obstet Gynecol. 1998; 179(3): 703-7.

10. Yen CF, Lin SL, Murk W, et al. Risk analysis of torsion and malignancy for adnexal masses during pregnancy. Fertil steril. 2009; 91(5): 1895-902.

11. Tailor A, Jurkovic D, Bourne TH, Collins WP, Campbell S. Sonographic prediction of malignancy in adnexal masses using multivariate logistic regression analysis. Ultrasound Obstet Gynecol. 1997; 10(1): 41-7.

12. Jacobs I, Oram D, Fairbanks J, Turner J, Frost C, Grudzinskas JG, et al. A risk of malignancy index incorporating CA 125 , ultrasound and menopausal status for the accurate preop- erative diagnosis of ovarian cancer. Br J obstet gynecol. 1990; 97(10): 922-9.

13. Lerner JP, Timor-Tritsch IE, Federman A, Abramovich G. Transvaginal ultrasonographic characterization of ovarian masses with an improved weighted scoring system. Am J Obstet Gynecol. 1994 Jan;170(1 Pt 1):81-5

14. Usui R, Minakami H, Kosuge S, Iwasaki R, Ohwada M, Sato I. A Retrospective Survey of Clinical, Pathologic and Prognostic Features of Adnexal Masses Operated On During Pregnancy. J Obstet Gynaecol Res. 2000; 26(2): 89-93.

15. Whitecar MP, Turner $S$ and Higby MK, Adnexal masses in pregnancy: a review of 130 cases undergoing surgical management. Am J Obstet Gynecol. 1999; 181(1): 19-24.

16. Sherard GB, Hodson CA, Williams HJ, Semer DA, Hadi HA and Tait DL. Adnexal masses and pregnancy: a 12-year experience. Am J Obstet Gynecol. 2003; 189(2): 358-62.

17. Türkçüoğlu I, Meydanli MM, Engin-Ustün $Y$, Ustün $Y$, Kafkasli A. Evaluation of histopathological features and pregnancy outcomes of pregnancy associated adnexal masses. J Obstet Gynaecol. 2009; 29(2): 107-9. 